\title{
Up-regulation of ceRNA TINCR by SP1 contributes to tumorigenesis in breast cancer
}

\author{
Yun Liu', Yaying $\mathrm{Du}^{2}$, Xiaopeng $\mathrm{Hu}^{2}$, Lu Zhao ${ }^{2}$ and Wenfei Xia ${ }^{2^{*}}$
}

\begin{abstract}
Background: Assembling evidences suggested that aberrant expression of tissue differentiation-inducing non-protein coding RNA (TINCR) intimately associated with variety of human cancer. However, the expression pattern and involvement of TINCR in breast cancer has not been fully investigated. Here we set out to analyze expression of TINCR in breast cancer and elucidate its mechanistic involvement in tumor incidence and progression.

Methods: The expression of TINCR was determined by q-PCR. SP1 binding sites were analyzed by ChIP-qPCR. The relative transcription activity was measured with luciferase reporter assay. Cell viability was measured with CCK-8 method. Clonogenic capacity was evaluated by soft agar assay. Cell apoptosis was analyzed by Annexin V/7-AAD staining. The migration and invasion were determined by trans-well assay and wound healing. The tumor growth in vivo was evaluated in xenograft mice model. Protein expression was quantified by immunoblotting.

Results: TINCR was aberrantly up-regulated by SP1, which in turn stimulated cell proliferation, anchorage-independent growth and suppressed cell apoptosis in breast cancer. TINCR silencing significantly suppressed migration and invasion in vitro and xenograft tumor growth in vivo. Mechanistically, TINCR modulated KLF4 expression via competing with miR-7, which consequently contributed to its oncogenic potential. MiR-7 inhibition severely compromised TINCR silencing-elicited tumor repressive effects.
\end{abstract}

Conclusion: Our data uncovered a crucial role of TINCR-miR-7-KLF4 axis in human breast cancer.

Keywords: TINCR, miR-7, KLF4, Breast cancer, Long non-coding RNA

\section{Background}

Breast cancer is one of the most common malignancies in women [1]. In 2016, approximately 246,660 new cases have been diagnosed with this disease and 40,450 cancer related deaths were claimed in US [2]. Risk factors intimately associate with breast cancer incidence include female gender, obesity, never giving a birth, hormone replacement therapy for menopause et al. [3]. In addition, the environmental exposure to ionizing radiation and unhealthy life style such as lack of exercise and excessive consumption of alcohol causally link to breast cancer as well [4]. Only about $5 \sim 10 \%$ of all cases are due to genetic disorders such as BRCA1 and BRCA2 mutations characterized

\footnotetext{
* Correspondence: huzhenxi973hust@outlook.com

²Department of General Surgery, Tongji Hospital, Tongji Medical College, Huazhong University of Science and Technology, 1095 Jiefang Avenue, Wuhan 430030, People's Republic of China

Full list of author information is available at the end of the article
}

in majority of hereditary breast-ovarian cancer syndrome [5]. With advances in our knowledge about this disease at molecular level, breast cancer now is unambiguously classified into four subtypes including Luminal $\mathrm{A}^{+}\left(\mathrm{ER}^{+} /\right.$ $\mathrm{PR}^{+} / \mathrm{HER} 2^{-}$, grade 1 or grade 2$)$, Luminal $\mathrm{B}\left(\mathrm{ER}^{+} / \mathrm{PR}^{+} /\right.$ HER2 ${ }^{+}$, grade 3), HER2 overexpression $\left(\mathrm{ER}^{-} / \mathrm{PR}^{-} /\right.$ $\mathrm{HER}^{+}$) and Triple Negative Breast Cancer (TNBC, $\mathrm{ER}^{-} /$ $\mathrm{PR}^{-} / \mathrm{HER}^{-}{ }^{-}$[6]. The clinical management of breast cancer essentially depends on variety of disease factors including tumor stage, age and genetic causes [7]. Surgery is the most efficient option for radically curative purpose in those early-diagnosed patients, which could be more favorable while in combination with chemotherapy or radiotherapy. Hormone blocking therapy is only applicable for hormone receptor positive patients [8]. As for many other hematological malignancies and solid tumors, cell checkpoint blockade-based immunotherapy is currently 
under intensive investigation for clinical application in breast cancer in view of its intrinsic strong immunogenicity [9].

It's estimated that $80 \%$ of human transcripts are not eventually translated into proteins, among which the long non-coding RNAs (lncRNA) are defined as class of RNA molecules longer than 200 nucleotides without protein-coding potential [10]. As of July 2017, there were 298 lncRNAs archived and functionally annotated in LncRNAdb (http://www.lncrnadb.org). The diverse biological functions of IncRNAs have been increasingly revealed that involved in multi-layered gene expression regulatory network [11]. In eukaryotes, IncRNAs target multiple cis- and trans- components of the transcription process, including the transcription activators, repressors, RNA polymerase II and even the DNA duplex [12]. In addition, lncRNAs also control various aspects of posttranscriptional mRNA processing in a similar way as microRNAs and snoRNAs, and potentially affect pre-mRNA splicing, transportation, translation and degradation [13]. Until recently, the crucial roles of lncRNAs in chromatin epigenetic modifications have been uncovered, which mediated imprinting, X-chromosome inactivation and telomere protections [14]. Tissue differentiation-inducing non-protein coding RNA (TINCR) is a spliced long noncoding RNA required for normal epidermal differentiation [15]. Assembling evidences suggested that aberrant expression of TINCR intimately associated with variety of human cancers [16, 17]. However, the expression pattern and involvement of TINCR in breast cancer has not been fully investigated. Here we set out to determine the expression status of TINCR and sought to elucidate its mechanistic linkage to breast cancer.

\section{Methods}

\section{Patient samples}

The protocol for human research was approved by the Ethics Committee of Tongji Hospital. Totally, 24 pairs of tumor (age 28-54) and adjacent normal samples were collected from the breast cancer patients enrolled in Tongji Hospital with written informed consent between March 2016 and September 2016. The diagnoses of breast cancer were histologically confirmed by three independent pathologists. The freshly collected samples were flash frozen in liquid nitrogen for RNA isolation.

\section{Cell culture}

The immortalized human mammary epithelial cell line MCF-10A and breast cancer cell lines MDA-MB-231, MDA-MB-435, MDA-MB-453, MDA-MB-468 and MCF-7 were obtained and authenticated by the America Typical Culture Collection (ATCC). All cells were cultured in RPMI-1640 supplemented with $10 \%$ fetal bovine serum and 1\% PSG (penicillin-streptavidin-glutamine). The
MCF-10A was additionally supplemented with $100 \mathrm{ng} / \mathrm{ml}$ cholera toxin. The log phase cells were maintained in $37{ }^{\circ} \mathrm{C}$ humidified incubator with $5 \% \mathrm{CO}_{2}$.

\section{Transfection}

The MCF-7 or MDA-MB-468 cells in log phase were seeded into 6-well plate the day before transfection. The indicated plasmids were transfected using Lipofectamine 2000 in strict accordance with the manufacturer's instructions. Transfection efficiency was evaluated by parallel assay with GFP and examined under inverted fluorescence microscope (Nikon).

\section{Real-time PCR}

The total RNA was extracted from indicated tissue samples or cell lines using Trizol reagent (Invitrogen) following the manufacturer's manual. The quality and quantity were determined by BioAnalyzer 2100 (Agilent) prior to further analysis. $1 \mu \mathrm{g}$ total RNA was reversely transcribed into cDNA by PrimeScript First Strand cDNA Synthesis Kit (Clontech) according to the manufacturer's instructions. The quantitative PCR was performed on ABI Prism 7900 HT. All primers used in this study were listed in Table 1.

\section{Chromatin immunoprecipitation (ChIP)}

ChIP assay was performed with the SimpleChIP Assay Kits (Cell Signaling Technology). Briefly, MCF-7 cells were first treated with $37 \%$ formaldehyde for $10 \mathrm{~min}$ at room temperature and subjected to ultrasonication on ice. The DNA-protein complex was immune-precipitated with SP1 antibody (Cell Signaling Technologies). The bound

Table 1 Primers used for qRT-PCR, qCHIP and siRNAs oligonucleotides

\begin{tabular}{ll}
\hline Primers used for qRT-PCR & \\
\hline TINCR-F & TGTGGCCCAAACTCAGGGATACAT \\
TINCR-R & AGATGACAGTGGCTGGAGTTGTCA \\
GAPDH-F & GCTCTCTGCTCCTCCTGTC \\
GAPDH-R & ACGACCAAATCCGTTGACTC \\
siRNAs/shRNA oligonucleotides & \\
scrambled & UUCUCCGAACGUGUCACGUdTdT \\
si-SP1-1 & CAGCGUUUCUGCAGCUACCUUGACU \\
si-SP1-2 & GACAGGUCAGUUGGCAGACUCUACA \\
siTINCR-1 & UAUUCCUUCAGCCAGUACCCAGGUC \\
siTINCR-2 & UUUCCAAGGUGGCACAGUGCUUUCC \\
qCHIP analysis of the TINCR promoter for SP1 ocCupanCy \\
BS3-F & TGACCTCGCTGATGGCTCT \\
BS3-R & TCAGGCGTCCGCTCCCCACT \\
BS1/2-F & TGAGGGGACCGTGGCA \\
BS1/2-R & TGGTAGCGCTTCCAGCGCGACA \\
\hline
\end{tabular}


DNA fragments were then reversely released and amplified by specific PRC reaction. The primers used in ChIP assay have been listed in Table 1 .

\section{Dual-luciferase reporter assay}

For SP1 induced overexpression of TINCR, wild-type, putative SP1 binding site deleted or mutated promoter regions were cloned into pGL4 plasmid. MCF-7 cells were transfected with luciferase reporter plasmid plus either SP1-expressing or empty vector (EV) by lipofectamine 2000. Cells were harvested $48 \mathrm{~h}$ later and relative luciferase activities were determined with Dual-luciferase Assay System (Promega, USA). For miR-7 regulated expression of TINCR, TINCR full length of transcript (wild-type, miR-7 target region deletion, miR-7 target region mutation) was fused to luciferase, and co-transfected MCF-7 or MDA-MB-468 cells with miR-7a/b. The luciferase activities were measured $48 \mathrm{~h}$ post-transfection.

\section{Immunoblotting}

The indicated cells were lysed in RIPA buffer on ice for $30 \mathrm{~min}$ and cell debris were discarded via refrigerated centrifugation (12,000 rpm for $15 \mathrm{~min})$. Equal amount of cell lysates was resolved by SDS-PAGE and then transferred to PVDF membrane in ice bath. The membrane was blocked with $5 \%$ milk in TBST buffer (0.05\% Tween-20), and incubated with primary antibody at $4{ }^{\circ} \mathrm{C}$ overnight. After wash with TBST, the membrane was incubated with HRP-labeled secondary antibody at room temperature for $1 \mathrm{~h}$. The protein bands were then visualized with enhanced chemiluminescence reagent (Cwbiotech).

\section{Cell proliferation assay}

The relative cell viability was measured using commercial available CCK-8 Kit (Dojindo). Equal number of cells with indicated treatment was seeded into 96-well plate in triplicate for $24 \mathrm{~h}$ culture. Then, $10 \mu \mathrm{l}$ of CCK- 8 solution was added into each well and incubated at $37^{\circ} \mathrm{C}$ for $1 \sim 4 \mathrm{~h}$. The OD450nm was recorded by microplate reader and cell viability was calculated.

\section{Soft agar assay}

MCF-7 and MDA-MB-468 were transfected with indicated plasmids. After $24 \mathrm{~h}$ culture, the cells were harvested and prepared into single-cell suspension in $2 \times$ RPMI 1640, and then mixed with equal volume of $0.6 \%$ low-melting-point agarose (Sigma). The mixture was laid on top of solidified layer of $0.6 \%$ agarose in serum-free growth medium. The fresh and complete culture medium was added for up to 2 weeks' culture. The visible colonies were stained with crystal violet and counted under light microscope.

\section{Cell apoptosis assay}

The exponentially growing cells were harvested and resuspended in HEPES buffer as single-cell solution. Staining with Annexin V-fluorescein isothiocyanate and 7-AAD (Sigma) was performed in dark at room temperature for $15 \mathrm{~min}$. The apoptotic cells were determined by flow cytometry (Beckman Coulter).

\section{Transwell assay}

The cell migration and invasion assay were performed with transwell chamber (BD). The indicated cells were starved for $24 \mathrm{~h}$ first, trypsinized and prepared into single-cell suspension in serum-free medium and laid on the top of polycarbonate Transwell filter (pre-coated with Matrigel (BD) for invasion assay). The lower compartment was supplied with complete culture medium containing $10 \%$ fetal bovine serum. $24 \mathrm{~h}$ later, the cells inside insert were completely removed with Q-tips. The migrated/invaded cells were fixed with $4 \%$ paraformaldehyde and stained with $0.025 \%$ crystal violet. For each group, the cell number was counted in five random fields under microscope to assess migratory/invasive capacity.

\section{Scratch healing assay}

MCF-7 and MDA-MB-468 cells were first transfected with either scramble or siTINCR in 6-well plate using Lipofectamine 2000. A straight line was drawn in each well using yellow tips to stimulate a wound. The scratch width was continuously monitored and recorded at $0,12,24$ and $36 \mathrm{~h}$ during healing process.

\section{Xenograft tumor}

Totally, 12 female BALB/c-nude mice (20-22 g, 4-6 weeks old) were obtained from the Shanghai Laboratory Animal Center (SLAC), and randomly divided into control and siRNA-TINCR groups after 1-week acclimatization. All experimental animals were housed in a pathogen-free environment and protocols were approved by the Committee of Animal Care and Use of Tongji Hospital. The single-cell solution was prepared and mixed with equal volume of Matrigel (BD Sciences) on ice. The mixture was cautiously inoculated subcutaneously into the right flanks of immunodeficiency mice. Tumor growth was monitored every week using and volume was determined with digital caliper according to the formula: TV $\left(\mathrm{mm}^{3}\right)=$ length $\times$ width $^{2} \times 0.5$. Mice were sacrificed at the endpoint indicated and subjected to macroscopic examination and weighing.

\section{Fluorescence in situ hybridization}

TINCR subcellular localization was determined by RNA hybridization technology performed with Stellaris FISH Probes (Human TINCR with CAL Fluor-Orange 560, Human GAPDH With Quasar 670 Dye, BioSearch 
Technology, CA, USA) in accordance with the manufacturer's instruction. The cells were counter-stained with DAPI and images were acquired with Zeiss LSM 800 confocal microscope.

\section{Statistical analysis}

All data in this study were obtained from at least three independent repeats unless specified. The statistical analysis was performed with SPSS 23 software and results were presented as Mean \pm standard deviation (SD). The one-way analysis of variance (ANOVA) was employed for statistical comparison. The $p$ value was calculated and $<0.05$ was considered as statistically different.

\section{Results}

\section{SP1 stimulated TINCR overexpression in human breast cancer}

The relative expression of TINCR was analyzed by q-PCR in 24 pairs of breast cancer tissue samples and corresponding adjacent normal tissues. As shown in Fig. 1a, TINCR was markedly over-expressed in breast cancer with 3 16-fold increase. We further determined the expression pattern of TINCR in panel of breast cancer cell lines. Consistent with the results from clinical samples, TINCR transcripts were significantly higher in cancer cell lines than in immortalized human mammary epithelial cell line MCF-10A (Fig. 1b). In line with previous investigations into other tumors $[16,17]$, our results from both clinical samples and cell lines demonstrated the consistent upregulation of TINCR in breast cancer. Moreover, the Kaplan-Meier cumulative survival curve showed more favorable prognosis in patients with low TINCR, which supported the tumorigenic roles of TINCR (Fig. 1c).

Next, we attempted to elucidate the potential regulatory mechanism underlying aberrant overexpression of TINCR in breast cancer. Close inspection of the promoter region of TINCR identified three putative SP1 binding sites (BSs) with high G/C content (Fig. 1d, upper pane). SP1 is a zinc finger transcription factor that recognizes and binds to the GC-rich motif in promoters of multiple genes, which physiologically involved in diverse cellular processes including differentiation, growth, apoptosis,

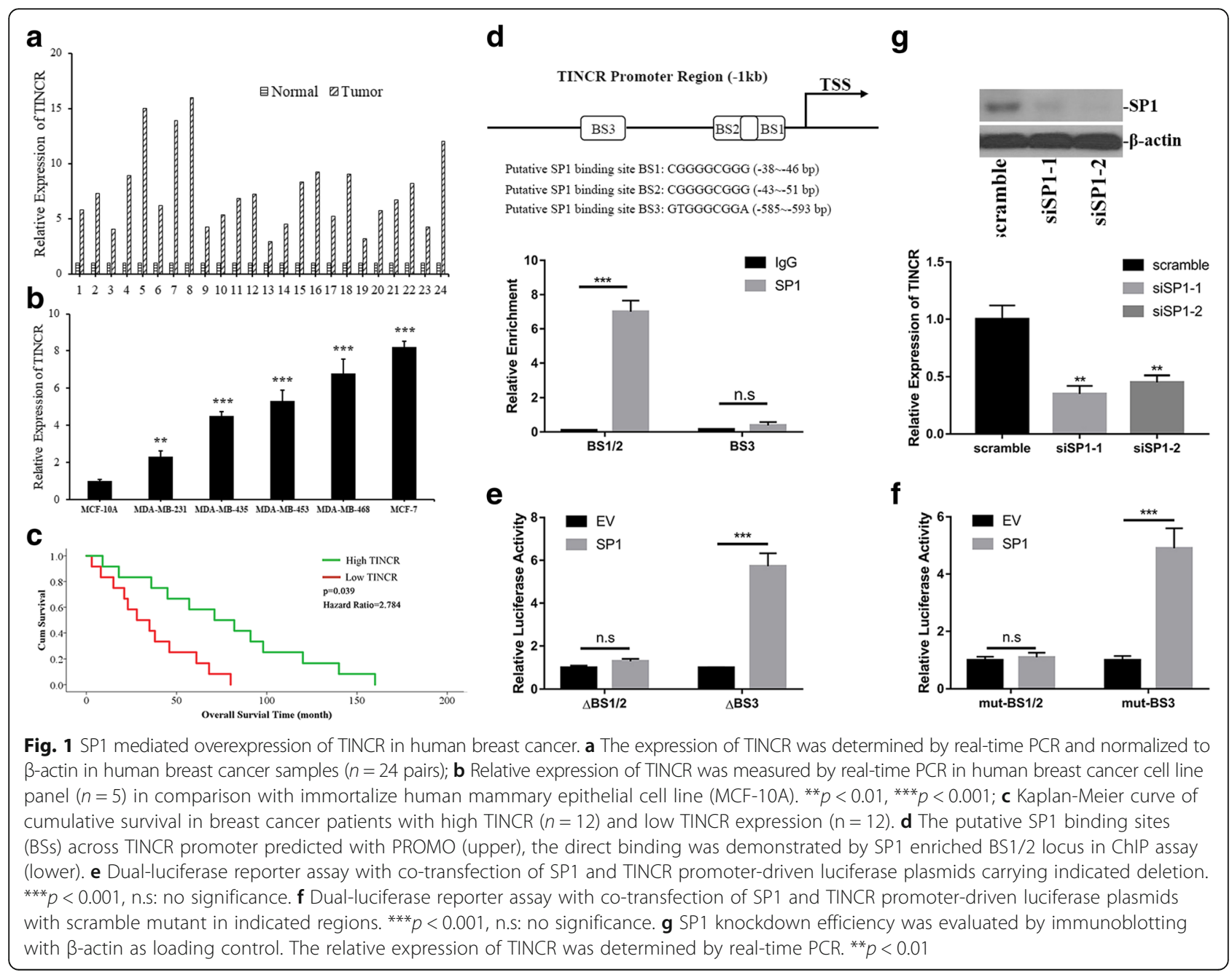


immune responses, DNA damage responses and chromatin remodeling [18]. SP1 functions as either activator or repressor of transcription via post-translational modifications such as phosphorylation, acetylation, glycosylation. Assembling evidences suggested that SP1 involved in cancer biology [19]. Here we first confirmed the direct binding of SP1 with suspected sites in TINCR promoter by ChIP assay. As shown in Fig. 1d lower pane, approximately 7 -fold increase of BS1/2 fragment was enriched in SP1 immunoprecipitate in comparison with IgG. However, no obvious enrichment of BS3 was detected in our results, which clearly suggested that BS1/2 were the cis- elements subjected to SP1 regulation. We further consolidated this phenomenon in TINCR promoter-driven luciferase reporter assay. Either deletion or mutation introduced to BS1/2 sites significantly compromised the SP1-stimulated transcription (Fig. 1e, f), while destruction in BS3 motif showed none of effects on luciferase activity. Furthermore, siRNAmediated knockdown of SP1 attenuated the endogenous expression of TINCR in MCF-7 cells (Fig. 1g). Taken together, our data demonstrated SP1 stimulated TINCR overexpression in breast cancer.

\section{TINCR knockdown inhibited malignant progression in breast cancer cells}

Next, we sought to understand the potential oncogenic role of TINCR in breast cancer. MCF-7 and MDA-MB468 cells were selected for mechanism study due to the relatively high expression of TINCR. SiRNA-mediated silencing of TINCR was first validated by quantitative PCR and around 70 80\% knockdown efficiencies were achieved in both cell lines (Fig. 2a). TINCR deficiency significantly suppressed cell proliferation in MDA-MB468 (Fig. 2b) and MCF-7 (Fig. 2c). In addition, the clonogenic capacity of MCF-7 and MDA-MB-468 was remarkably decreased in TINCR-deficient cells in comparison to the proficient counterparts (Fig. 2d, e). TINCR knockdown stimulated spontaneous apoptosis in both cell lines as well (Fig. 2f, g). In summary, our data demonstrated that inhibition of TINCR blocked malignant progression in breast cancer.

\section{TINCR silencing suppressed migration and invasion of breast cancer cells}

Our previous data demonstrated that TINCR knockdown significantly suppressed cell proliferation, anchorageindependent growth and stimulated spontaneous apoptosis in breast cancer cells. Next, we sought to determine its impact on migration and invasion of breast cancer cells. The results acquired from transwell assay demonstrated that TINCR-silencing remarkably suppressed migratory capacity of MCF-7 and MDA-MB-468 (Fig. 3a, b). Likewise, the number of invaded cells in Matrigel-coated transwell was decreased by TINCR knockdown (Fig. 3c, d). We have performed scratch healing assay as well to validate the compromised migration elicited by TINCR silencing. Consistent with transwell assay, the wound closure was decelerated in TINCR-deficient cells in comparison with control (Fig. 3e, f), which indicated the crucial role of TINCR in maintenance of cell migration in breast cancer. Taken together, our data demonstrated that TINCR apparently involved in the metastatic process during breast tumor progression.

\section{TINCR silencing suppressed tumor growth in vivo}

Noteworthily, all the above-mentioned data was acquired from cell lines in vitro. To exclude the potential artifacts associated with cell culture, we further investigated the impact on tumor growth of TINCR inhibition in xenograft tumor model. MDA-MB-468 cells transfected with either scramble or siTINCR were subcutaneously inoculated into nude mice. The continuous inspection observed significant delay in tumor growth in TINCR-knockdown mice compared to the control (Fig. 4a). The representative images of macroscopic xenograft tumor extracted from sacrificed mice revealed much smaller size in TINCR knockdown group (Fig. 4b). And the average weight of TINCR-deficient xenograft tumor was $0.5 \mathrm{~g}$ compared to $1.3 \mathrm{~g}$ in control mice (Fig. 4c). We verified the persistent knockdown of TINCR in our xenograft tumor model at the endpoint of our experiment (Fig. 4d). Therefore, we consolidated the observation that TINCR-knockdown significantly suppressed tumor growth both in vitro and in vivo.

\section{TINCR functioned as endogenous competing IncRNA of miR-7 and involved in KLF4 regulation}

The accumulative evidences suggested that long noncoding RNA might function as endogenous competing RNA against miRNAs. Here we sought to investigate the possible molecular events underlying oncogenic activity of TINCR along this direction. We employed LncRNABase online algorithm to predict the candidate miR with the potential to directly compete with TINCR, and identified miR-7 as the top one in the putative targets list. The alignment between TINCR and miR-7 was shown in Fig. 5a. Range of investigations have uncovered the important roles of miR-7 in human malignancies with increased list of target genes have been identified [20-27]. In line with previous observations, the direct binding of miR-7 with TINCR was experimentally validated in our luciferase reporter assay. The exogenous introduction of miR-7 caused about $75 \%$ reduction in MDA-MB-468 and $60 \%$ reduction in MCF-7 of TINCR-fused luciferase activity (Fig. 5b, c). We further experimentally validated the putative binding sites of miR-7 on TINCR transcript. Either deletion or mutation introduced into the 


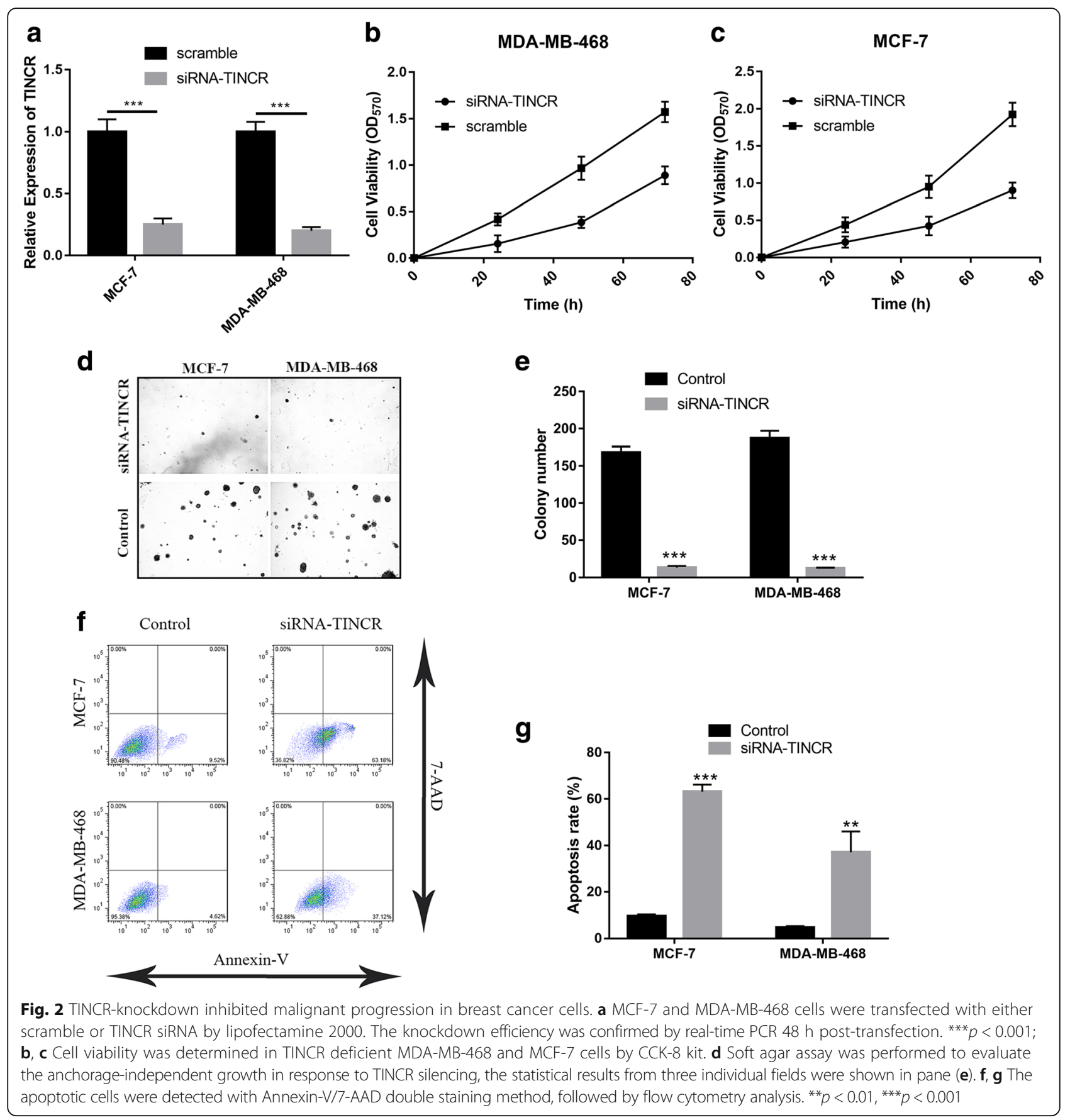

suspected regions of TINCR abolished miR-7-inhibited luciferase activities (Fig. 5b, c, d, e). Consistent with its physiological role as endogenous competing RNA, the RNA hybridization results clearly demonstrated the predominant localization of TINCR in cytoplasm (Additional file 1: Figure S1). Taken together, our data suggested that TINCR might function as molecular sponge of miR-7, which eventually contributed to its oncogenic activity.
Kruppel Like Factor 4 (KLF4) is a zinc finger protein with physiological function as transcription factor. Several studies implied that KLF4 was the potential target of miR-7. Along this line, here we further investigated the potential that TINCR modulated KLF4 expression via competing with miR-7. The putative target site in 3'UTR region of KLF4 aligned with miR-7 seed region based on the micrRNA.org online prediction was showed in Fig. 5f. Exogenous introduction of miR-7a/b remarkably inhibited 

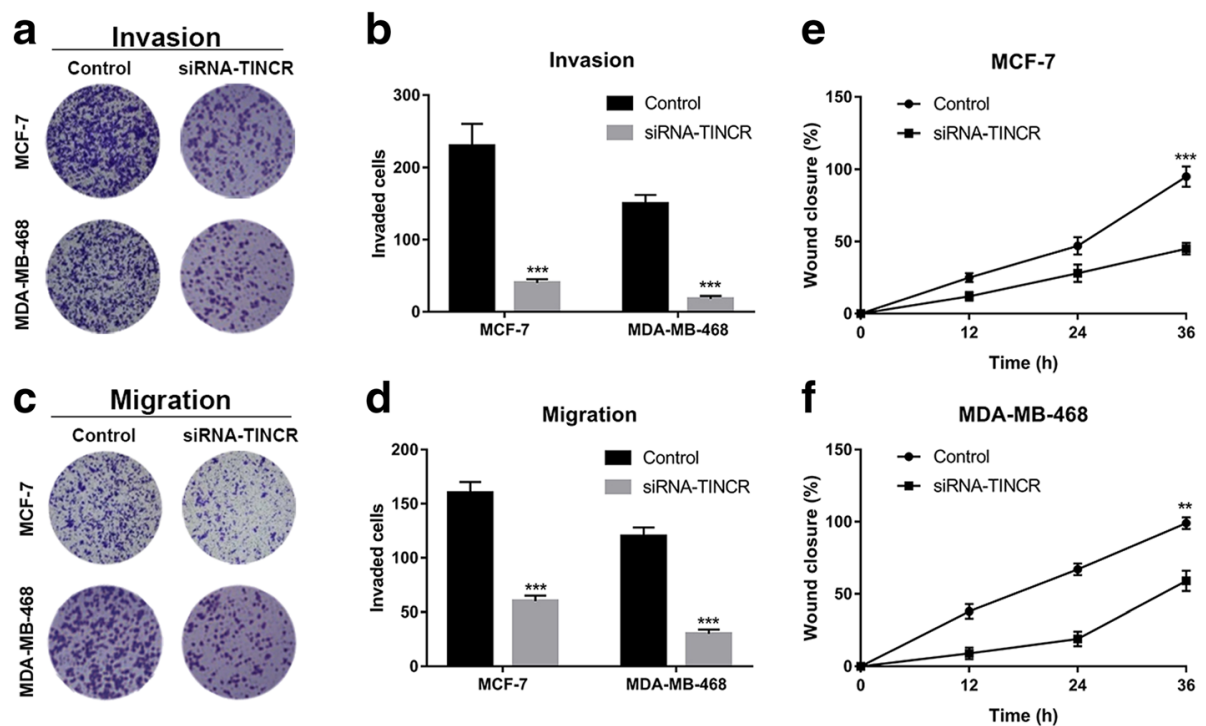

Fig. 3 TINCR-silencing suppressed migration and invasion of breast cancer cells. $\mathbf{a}$, $\mathbf{b}$ The invasive capacity of indicated cells were determined by Matrigel coated transwell assay. The representative images were shown in pane (a) and the average counting results in pane (b). ${ }^{* * *} p<0.001$. c, $\mathbf{d}$ The migratory capacities were evaluated by transwell migration assay. The representative images were shown in pane (c) and the average counting results in pane $(\mathbf{d}) .{ }^{* * *} p<0.001$. e, $\mathbf{f}$ Scratch-healing assay was performed to assess migration in TINCR deficient MCF-7 and MDA-MB-468 cells. The wound closure was monitored at 0,12, 24 and $36 \mathrm{~h}$ respectively

endogenous KLF4 in our immunoblotting results in both MCF-7 and MDA-MB-468 cells. This inhibitory effect was readily reversed by co-introduction of either TINCR or miR-7 specific inhibitor (Fig. 5g). The regulation of KLF4 by TINCR was evaluated at transcription level as well. Consistently, the transcripts of KLF4 were decreased in response to $\mathrm{miR}-7 \mathrm{a} / \mathrm{b}$, and subsequently restored by ectopic TINCR or miR-7 inhibitor treatment (Fig. 5h). The expression status of SP1-TINCR-miR-7-KLF4 was further characterized in xenograft tumor at both transcriptional and translational level (Fig. 5i), which definitely consolidated our in vitro observations.

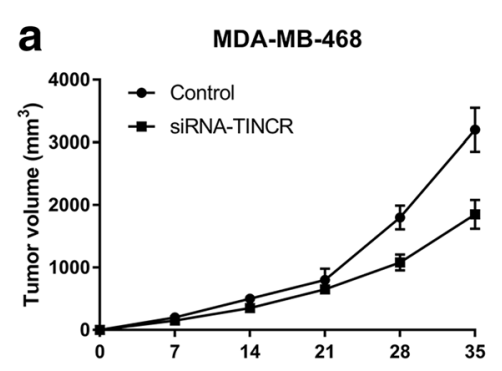

\section{b}
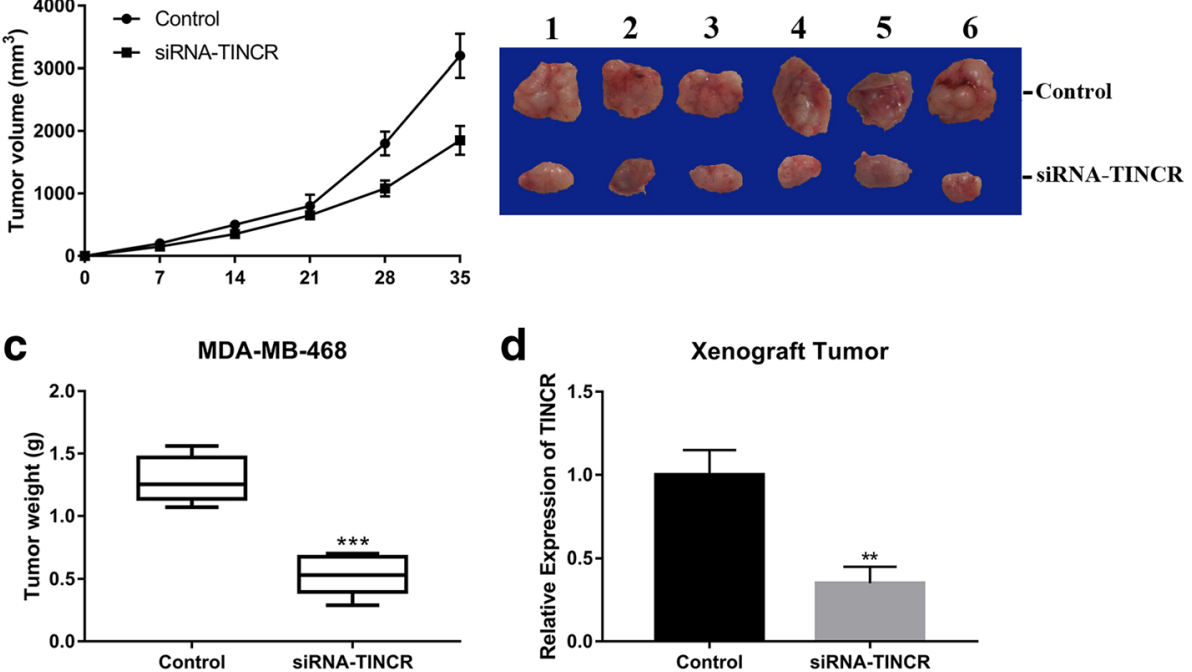

d

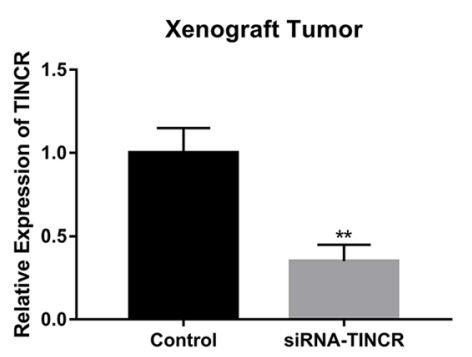

Fig. 4 TINCR-silencing suppressed tumor growth in vivo. a MDA-MB-468 cells transfected with either control or siRNA-TINCR were subcutaneously inoculated into nude mice. The tumor volume was measured at $0,7,14,21,28$ and 35 days post-injection respectively. $\mathbf{b}$ The macroscopic images of representative xenograft tumor 5 weeks post-inoculation. $\mathbf{c}$ The tumor weight was measured after tumor-bearing mice were sacrificed. ${ }^{* * *} p<0.001$. d The persistent silencing of TINCR in tumor was confirmed by real-time PCR. ${ }^{* *} p<0.01$ 


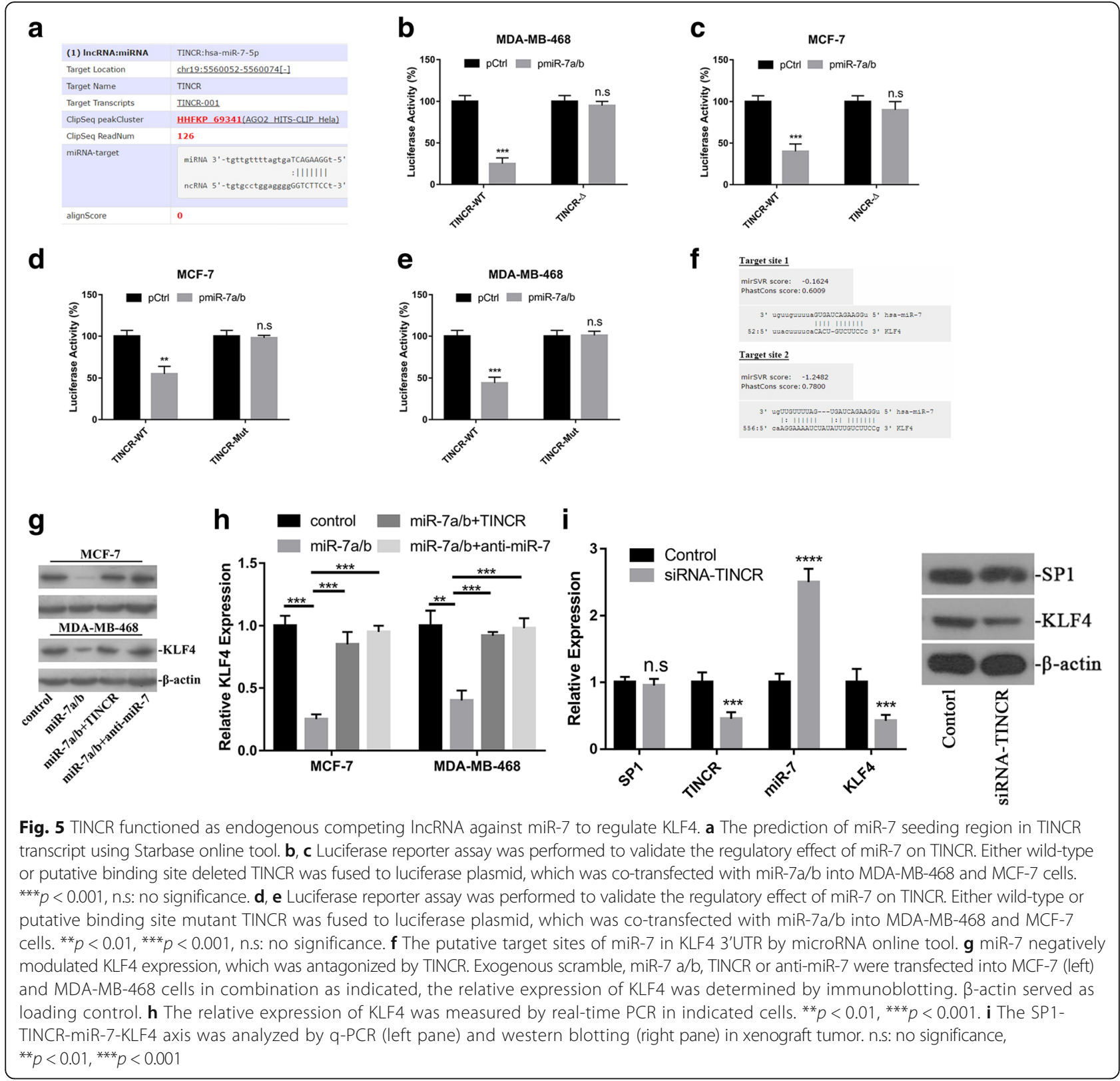

miR-7 inhibition abrogated TINCR-silencing elicited tumor suppressive effect

Our previous data suggested that TINCR functioned as competing endogenous RNA (ceRNA) to compete with miR-7 in regulation of KLF4. Next, we sought to determine the extent that this regulatory axis was involved in the oncogenic activity of TINCR in breast cancer. The endogenous expression of miR-7 in response to TINCR knockdown was analyzed in both MCF-7 and MDAMB-468 cells. As shown in Fig. 6a, TINCR-silencing induced more than 3-fold increase of miR-7, which was completely abrogated by co-treatment with miR-7 inhibitor. Consistent with previous results, TINCR-knockdown significantly suppressed breast cancer cell proliferation, while simultaneous inhibition of miR-7 in this setting almost abolished this effect (Fig. 6b, c). Similarly, the reduction in colony formation capacity elicited by TINCR silencing was readily restored while miR-7 was specifically inhibited (Fig. 6d). The inhibitory effects on invasive behavior of breast cancer cells imposed by TINCRknockdown was almost abrogated as well (Fig. 6e). Our data unambiguously demonstrated that miR-7 predominately involved in oncogenic activity of TINCR in breast cancer.

\section{Discussion}

TINCR is a critical modulator required for normal epidermal differentiation via regulation of array of genes 


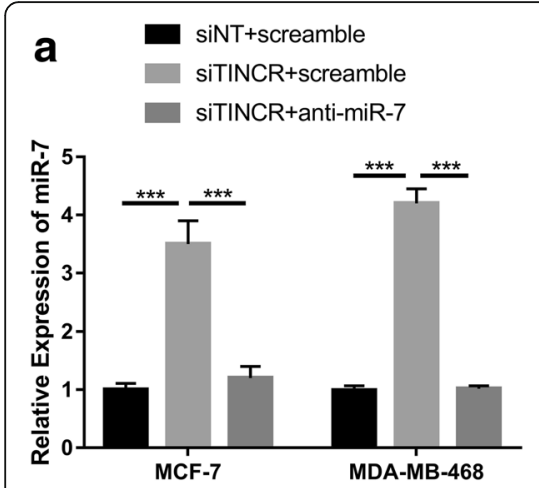

b

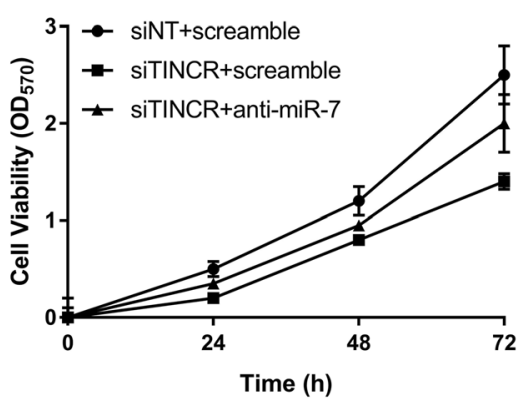

C

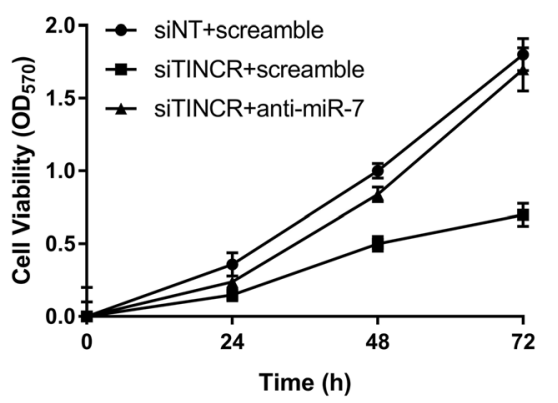

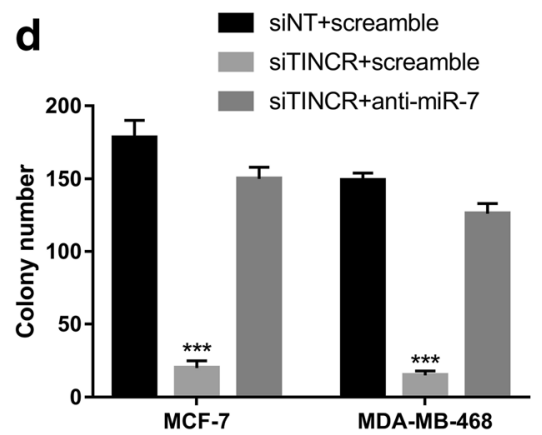

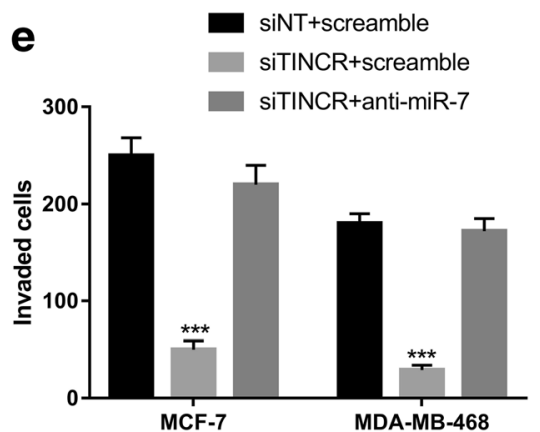

Fig. 6 miR-7-inhibition abrogated TINCR-silencing elicited tumor suppressive effect. a The relative expression of miR-7 in response to TINCR knockdown or miR-7 specific inhibitor was determined by real-time PCR in both MCF-7 and MDA-MB-468 cells. ${ }^{* * *} p<0.001$. b, c The suppressive effect on cell proliferation elicited by TINCR knockdown was relieved by miR-7 inhibition. The cell viability was monitor at 0, 24, 48 and $72 \mathrm{~h}$ respectively using CCK-8 assay. $\mathbf{d}$ Colony formation assay was performed to determine the anchorage-independent growth of indicated cells. ${ }^{* * *} p<0.001$. e The invasive capacity was assessed by Matrigel coated Transwell assay. ${ }^{* * *} p<0.001$

involving in this physiological process [28]. More recently, the potential roles of TINCR in tumor biology have been cumulatively uncovered in variety of human malignances. For instance, $\mathrm{Xu}$ et al. demonstrated that E2F1 stimulated TINCR/STAU1/CDKN2B signaling axis exacerbated gastric cancer progression [16]. Zheng et al. proposed that genetic variation of TINCR contributed to the susceptibility and progression of colorectal cancer [29]. A genome-wide lncRNA microarray profiling performed by Zhang et al. has identified circulating TINCR as molecular marker for gastric cancer [30]. Xu et al. reported that SP1-induced upregulation of TINCR regulating apoptosis by affecting KLF2 mRNA stability in gastric cancer [17]. In esophageal squamous cell carcinoma, TINCR was reported to be up-regulated and promote tumorigenesis as well [31]. However, another study from $\mathrm{Li}$ group displayed the anti-tumor activity of TINCR in colorectal cancer, wherein loss of TINCR expression promoted proliferation, metastasis through activating EpCAM cleavage [32]. Until now, the expression and potential linkage to tumorigenesis of TINCR in breast cancer has not been fully investigated. Noteworthily, while we prepared our manuscript, Xu et al. identified the oncogenic TINCR along with a cluster of lncRNAs were upregulated in breast cancer via analysis of GEO and TCGA databases [33], which definitely consolidated our finding.

Here we for the first time demonstrated overexpression of TINCR in both breast cancer tissues and cell lines. High level of TINCR in breast cancer patients significantly associated with relatively poor prognosis. Moreover, with the aid of bioinformatics tool, here we proposed that SP1 specifically modulated TINCR expression via direct binding to its promoter region. We further experimentally validated the putative cis- elements for SP1 in TINCR. TINCR silencing significantly inhibited cell proliferation, anchorage-independent growth, and most notably, stimulated simultaneous apoptosis in vitro. We also evaluated the potential contribution of TINCR to breast tumor metastasis. In our transwell and scratch healing assays, TINCR-knockdown remarkably compromised the migratory and invasive capacity. The pro-tumor activity of TINCR was further demonstrated in xenograft tumor mouse model.

Assembling evidences suggested that IncRNA could function as ceRNA to sponge miRs in either physiological or pathological conditions, especially in variety of human cancers [34]. For example, lncRNA-PVT1 promoted 
pancreatic cancer cells proliferation and migration through acting as a molecular sponge to regulate miR448 [35]. TP73-AS1 has been demonstrated to promote breast cancer proliferation through miR-200a-mediated TFAM inhibition [36]. Intriguingly, an integrated analysis of long non-coding RNA competing interactions in 361 gastric cancer indicated that TINCR might function as ceRNA with multiple potential miRNAs [37]. In line with this notion, in this study we performed bioinformatic analysis to identify miR-7 as one of TINCR targets. Our data further experimentally confirmed the direct interaction between miR-7 and TINCR, which underlaid its competitive regulation of miR-7 target genes.

MiR-7 is conventionally considered as a tumor suppressor miRNA in variety of human malignancies including breast cancer [24], brain cancer [21], liver cancer [38], colon cancer [26]. However, the opposite conclusion emerged as well in especially lung cancer [39], suggested both oncogene and tumor suppressor roles of miR-7 probably in an organ context-dependent manner. The candidate target genes of miR-7 involving in tumor biology have been extensively identified and systematically reviewed by $\mathrm{Gu}$ et al. [20]. For instance, miR-7 has been demonstrated to function as a tumor-suppressor gene in pancreatic carcinoma via regulation of ILF2 [40]. MiR-7 also suppressed cell proliferation and induced apoptosis of breast cancer cells predominately by targeting REGy [25]. Moreover, miR-7 was shown to arrest cell cycle in G1 phase by directly targeting CCNE1 in human hepatocellular carcinoma cells [27]. Most notably, several studies indicated that miR-7 functioned as tumor suppressor gene via direct regulation of KLF4. Okuda et al. reported that miR-7 capable of suppressing brain metastasis of breast cancer stem-like cells by modulating KLF4 [23]. Chang et al. reported that miR-7 inhibited the tumorigenesis and stemness of prostate cancer via repressing KLF4/ PI3K/Akt/p21 pathway [41]. On the other hand, inhibition of miR-7 was shown to promote angiogenesis in human umbilical vein endothelial cells by up-regulating VEGF via KLF4 [22]. Consistent with these observations, our data suggested that inhibitory effect on KLF4 expression of miR-7 was significantly mitigated by TINCR in breast cancer via competitive mechanism.

In summary, in this study we characterized TINCR overexpression regulated by SP1 transcription factor. High level of TINCR in turn competed with miR-7, and stabilized and promoted KLF4 expression, which consequently contributed to the oncogenic activity of TINCR. Our results clearly demonstrated the critical role of TINCR in tumorigenesis and metastasis-related malignant behavior in breast cancer, and predominant role of miR-7 in mediating this effect. Most importantly, our data suggested that either specific inhibition of TINCR or complement with miR-7 likely held great promise for breast cancer therapeutics.

\section{Conclusions}

Our data suggested a crucial role of TINCR-miR-7-KLF4 axis in human breast cancer and up-regulation of ceRNA TINCR by SP1 contributes to tumorigenesis in breast cancer.

\section{Additional file}

Additional file 1: Figure S1 TINCR predominantly located in cytoplasm. Subcellular localization of TINCR was analyzed RNA hybridization with the specific Stellaris RNA FISH probes followed by confocal microscope imaging. TINCR was detected in red channel, while cytoplasmic GAPDH transcript was detected in green channel. The nuclei were counter-stained with DAPI. (JPEG 29 kb)

\section{Abbreviations}

CeRNAs: Competing endogenous RNAs; ChIP: Chromatin immunoprecipitation; KLF4: Kruppel Like Factor 4; LncRNA: Long non-coding RNAs; ATCC: America Typical Culture Collection; PSG: Penicillin-streptavidin-glutamine; TINCR: Tissue differentiation-inducing non-protein coding RNA

\section{Acknowledgements}

Not applicable

Funding

Not applicable.

\section{Availability of data and materials}

All data generated or analyzed during this study are included in this published article.

\section{Authors' contributions}

XWF conceived of the strategies and designed the study; LY, DYY, HXP and $\mathrm{ZL}$ conducted experiments and analysed data; XWF and LY wrote the manuscript. All authors have read and approved the final version of this manuscript.

\section{Ethics approval and consent to participate}

The tumor and adjacent normal tissue samples were collected from Tongji Hospital with written informed consents. The experimental protocol was approved by the Ethics Committee of Tongji Hospital, Tongji Medical College, Huazhong University of Science and Technology.

Consent for publication

Not applicable.

\section{Competing interests}

The authors declare that they have no competing interests.

\section{Publisher's Note}

Springer Nature remains neutral with regard to jurisdictional claims in published maps and institutional affiliations.

\section{Author details}

'Department of ENT, Tongji Hospital, Tongji Medical College, Huazhong University of Science and Technology, 1095 Jiefang Avenue, Wuhan 430030, People's Republic of China. 'Department of General Surgery, Tongji Hospital, Tongji Medical College, Huazhong University of Science and Technology, 1095 Jiefang Avenue, Wuhan 430030, People's Republic of China.

Received: 21 August 2017 Accepted: 20 March 2018

Published online: 03 April 2018

\section{References}

1. Harbeck N, Gnant M. Breast cancer. Lancet. 2017;389(10074):1134-50.

2. Siegel RL, Miller KD, Jemal A. Cancer statistics, 2017. CA Cancer J Clin. 2017;67(1):7-30. 
3. Peairs KS, Choi Y, Stewart RW, Sateia HF. Screening for breast cancer. Semin Oncol. 2017;44(1):60-72.

4. Cao Y, Giovannucci EL. Alcohol as a risk factor for Cancer. Semin Oncol Nurs. 2016;32(3):325-31.

5. Nik-Zainal S, Davies H, Staaf J, Ramakrishna M, Glodzik D, Zou X, Martincorena I, Alexandrov LB, Martin S, Wedge DC, et al. Landscape of somatic mutations in 560 breast cancer whole-genome sequences. Nature. 2016;534(7605):47-54.

6. Cancer Genome Atlas N. Comprehensive molecular portraits of human breast tumours. Nature. 2012;490(7418):61-70.

7. Saini KS, Taylor C, Ramirez AJ, Palmieri C, Gunnarsson U, Schmoll HJ, Dolci SM, Ghenne C, Metzger-Filho O, Skrzypski M, et al. Role of the multidisciplinary team in breast cancer management: results from a large international survey involving 39 countries. Ann Oncol. 2012;23(4):853-9.

8. Vilquin P, Cohen P, Maudelonde T, Tredan O, Treilleux I, Bachelot T, Heude $P E$. New therapeutical strategies in metastatic hormone-dependent breast cancer. Bull Cancer. 2015;102(4):367-80.

9. Pusztai L, Karn T, Safonov A, Abu-Khalaf MM, Bianchini G. New strategies in breast Cancer: immunotherapy. Clin Cancer Res. 2016;22(9):2105-10.

10. Perkel JM. Visiting "noncodarnia". BioTechniques. 2013;54(6):301. 303-304

11. Mercer TR, Dinger ME, Mattick JS. Long non-coding RNAs: insights into functions. Nat Rev Genet. 2009;10(3):155-9.

12. Goodrich JA, Kugel JF. Non-coding-RNA regulators of RNA polymerase II transcription. Nat Rev Mol Cell Biol. 2006;7(8):612-6.

13. Engreitz JM, Haines JE, Perez EM, Munson G, Chen J, Kane M, McDonel PE, Guttman M, Lander ES. Local regulation of gene expression by IncRNA promoters, transcription and splicing. Nature. 2016;539(7629):452-5.

14. Sanchez-Elsner T, Gou D, Kremmer E, Sauer F. Noncoding RNAs of trithorax response elements recruit Drosophila Ash1 to Ultrabithorax. Science. 2006;311(5764):1118-23.

15. Kretz M. TINCR, staufen1, and cellular differentiation. RNA Biol. 2013;10(10):1597-601

16. Xu TP, Wang YF, Xiong WL, Ma P, Wang WY, Chen WM, Huang MD, Xia R, Wang $R$, Zhang EB, et al. E2F1 induces TINCR transcriptional activity and accelerates gastric cancer progression via activation of TINCR/STAU1/CDKN2B signaling axis. Cell Death Dis. 2017;8(6):e2837.

17. Xu TP, Liu XX, Xia R, Yin L, Kong R, Chen WM, Huang MD, Shu YQ. SP1induced upregulation of the long noncoding RNA TINCR regulates cell proliferation and apoptosis by affecting KLF2 mRNA stability in gastric cancer. Oncogene. 2015;34(45):5648-61.

18. Beishline K, Azizkhan-Clifford J. Sp1 and the 'hallmarks of cancer'. FEBS J. 2015;282(2):224-58.

19. Chu S. Transcriptional regulation by post-transcriptional modification-role of phosphorylation in Sp1 transcriptional activity. Gene. 2012;508(1):1-8.

20. Kalinowski FC, Brown RA, Ganda C, Giles KM, Epis MR, Horsham J, Leedman PJ. microRNA-7: a tumor suppressor miRNA with therapeutic potential. Int J Biochem Cell Biol. 2014;54:312-7.

21. Kefas B, Godlewski J, Comeau L, Li Y, Abounader R, Hawkinson M, Lee J, Fine $\mathrm{H}$, Chiocca EA, Lawler S, et al. microRNA-7 inhibits the epidermal growth factor receptor and the Akt pathway and is down-regulated in glioblastoma. Cancer Res. 2008;68(10):3566-72.

22. Li YZ, Wen L, Wei X, Wang QR, Xu LW, Zhang HM, Liu WC. Inhibition of miR7 promotes angiogenesis in human umbilical vein endothelial cells by upregulating VEGF via KLF4. Oncol Rep. 2016;36(3):1569-75.

23. Okuda $H$, Xing F, Pandey PR, Sharma S, Watabe M, Pai SK, Mo YY, liizumiGairani M, Hirota S, Liu Y, et al. miR-7 suppresses brain metastasis of breast cancer stem-like cells by modulating KLF4. Cancer Res. 2013;73(4):1434-44.

24. Reddy SD, Ohshiro K, Rayala SK, Kumar R. MicroRNA-7, a homeobox D10 target, inhibits p21-activated kinase 1 and regulates its functions. Cancer Res. 2008;68(20):8195-200.

25. Shi Y, Luo X, Li P, Tan J, Wang X, Xiang T, Ren G. miR-7-5p suppresses cell proliferation and induces apoptosis of breast cancer cells mainly by targeting REGgamma. Cancer Lett. 2015;358(1):27-36.

26. Zhang N, Li X, Wu CW, Dong Y, Cai M, Mok MT, Wang H, Chen J, Ng SS, Chen $\mathrm{M}$, et al. microRNA-7 is a novel inhibitor of YY1 contributing to colorectal tumorigenesis. Oncogene. 2013;32(42):5078-88.

27. Zhang $X$, Hu S, Zhang $X$, Wang L, Zhang $X$, Yan B, Zhao J, Yang A, Zhang R. MicroRNA-7 arrests cell cycle in G1 phase by directly targeting CCNE1 in human hepatocellular carcinoma cells. Biochem Biophys Res Commun. 2014;443(3):1078-84.
28. Kretz M, Siprashvili Z, Chu C, Webster DE, Zehnder A, Qu K, lee CS, Flockhart RJ, Groff AF, chow J, et al. control of somatic tissue differentiation by the long non-coding RNA TINCR. Nature. 2013;493(7431):231-5.

29. Zheng Y, Yang C, Tong S, Ding Y, Deng W, Song D, Xiao K. Genetic variation of long non-coding RNA TINCR contribute to the susceptibility and progression of colorectal cancer. Oncotarget. 2017;8(20):33536-43.

30. Zhang K, Shi H, Xi H, Wu X, Cui J, Gao Y, Liang W, Hu C, Liu Y, Li J, et al. Genome-wide IncRNA microarray profiling identifies novel circulating IncRNAs for detection of gastric Cancer. Theranostics. 2017;7(1):213-27.

31. Xu Y, Qiu M, Chen Y, Wang J, Xia W, Mao Q, Yang L, Li M, Jiang F, Xu L, et al. Long noncoding RNA, tissue differentiation-inducing nonprotein coding RNA is upregulated and promotes development of esophageal squamous cell carcinoma. Dis Esophagus. 2016;29(8):950-8.

32. Zhang ZY, Lu YX, Zhang ZY, Chang YY, Zheng L, yuan L, Zhang F, Hu YH, Zhang WJ, li XN. loss of TINCR expression promotes proliferation, metastasis through activating EPCAM cleavage in colorectal cancer. Oncotarget. 2016;7(16):22639-49.

33. Xu S, Kong D, Chen Q, Ping Y, Pang D. Oncogenic long noncoding RNA landscape in breast cancer. Mol Cancer. 2017;16(1):129.

34. Karreth FA, Pandolfi PP. ceRNA cross-talk in cancer: when ce-bling rivalries go awry. Cancer Discov. 2013;3(10):1113-21.

35. Zhao L, Kong H, Sun H, Chen Z, Chen B, Zhou M. LncRNA-PVT1 promotes pancreatic cancer cells proliferation and migration through acting as a molecular sponge to regulate miR-448. J Cell Physiol. 2017;233(5):4044-55.

36. Yao J, Xu F, Zhang D, Yi W, Chen X, Chen G, Zhou E. TP73-AS1 promotes breast cancer cell proliferation through miR-200a-mediated TFAM inhibition. J Cell Biochem. 2017;119(1):680-90.

37. Li CY, Liang GY, Yao WZ, Sui J, Shen X, Zhang YQ, Peng H, Hong WW, Ye $Y C$, Zhang ZY, et al. Integrated analysis of long non-coding RNA competing interactions reveals the potential role in progression of human gastric cancer. Int J Oncol. 2016;48(5):1965-76.

38. Ning BF, Ding J, Liu J, Yin C, Xu WP, Cong WM, Zhang Q, Chen F, Han T, Deng $X$, et al. Hepatocyte nuclear factor 4 alpha-nuclear factor-kappaB feedback circuit modulates liver cancer progression. Hepatology. 2014;60(5):1607-19.

39. Cao Q, Mao ZD, Shi YJ, Chen Y, Sun Y, Zhang Q, Song L, Peng LP. MicroRNA-7 inhibits cell proliferation, migration and invasion in human non-small cell lung cancer cells by targeting FAK through ERK/MAPK signaling pathway. Oncotarget. 2016;7(47):77468-81.

40. Bi Y, Shen W, Min M, Liu Y. MicroRNA-7 functions as a tumor-suppressor gene by regulating ILF2 in pancreatic carcinoma. Int J Mol Med. 2017;39(4):900-6.

41. Chang YL, Zhou PJ, Wei L, Li W, Ji Z, Fang YX, Gao WQ. MicroRNA-7 inhibits the stemness of prostate cancer stem-like cells and tumorigenesis by repressing KLF4/PI3K/Akt/p21 pathway. Oncotarget. 2015;6(27):24017-31.

\section{Submit your next manuscript to BioMed Central and we will help you at every step:}

- We accept pre-submission inquiries

- Our selector tool helps you to find the most relevant journal

- We provide round the clock customer support

- Convenient online submission

- Thorough peer review

- Inclusion in PubMed and all major indexing services

- Maximum visibility for your research

Submit your manuscript at www.biomedcentral.com/submit
Biomed Central 